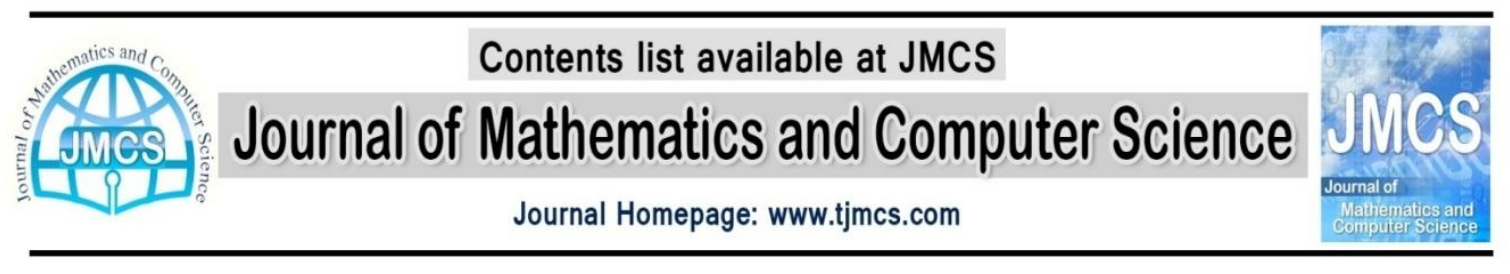

\title{
Fuzzy Prime and Anti Fuzzy Prime Implicative Filters of Lattice Wajsberg Algebras
}

\author{
Basheer Ahamed Mohideen* and A. Ibrahim** \\ *Department of Mathematics, Faculty of science, University of Tabuk, \\ Tabuk, Kingdom of Saudi Arabia. \\ **Department of Mathematics, Texcity Arts \& Science College, Coimbatore -641105, \\ Tamilnadu, India. \\ *Email: bahamed@ut.edu.sa; dr.basheer09@gmail.com \\ **Email: ibrahimaadhil@yahoo.com
}

Article history:

Received July 2014

Accepted September 2014

\begin{abstract}
In this paper, we introduce the concepts of a fuzzy prime and an anti fuzzy prime implicative filter of lattice $\mathrm{W}$ - algebras and discuss some of their properties.
\end{abstract}

Keywords: Wajsberg algebras, Implicative filters, Prime implicative filters, Fuzzy prime implicative filters, Anti fuzzy prime filters

Mathematical subject Classification 2010: 03E72, 03G25, 06F35

\section{Introduction}

The concept of fuzzy set was first introduced by Zadeh [8] in 1965, since then these ideas have been applied to other algebraic structures such as semigroups, groups, rings, modules, vector spaces and topologies. The importance of such algebraic structures can be seen from the latest research, which has been carried out in the last few years. In 1958, Rose et al [6] introduced the notion of Wajsberg algebras. Font et al [3] introduced the definition of implicative filters and family of implicative filters in lattice $\mathrm{W}$-algebra and investigated some of their properties. At present, fuzzification concepts have been applied to other algebraic structures such as groups, rings, and so on. In 1991, Xi [7] applied the concept of fuzzy sets to $B C K$-algebras that are introduced by Imai and Is'eki [5]. Basheer et al [1,2] introduced the notion of a fuzzy implicative filter of lattice Wajsberg algebras; an anti fuzzy implicative filter of lattice W-algebras, and investigated some of their properties. Recently, Eshagh Hosseinpour [4] discussed TRough Fuzzy Subgroups of Groups. In this paper, we introduce the notions of fuzzy prime and anti fuzzy prime implicative filters, further we discuss some related properties of fuzzy prime and anti fuzzy prime filters with illustrations. Finally, we describe some results of fuzzy prime and anti fuzzy implicative filter in lattice $\mathrm{W}$ - algebras. 
B. A. Mohideen, A. Ibrahim / J. Math. Computer Sci. 9 (2014), 25-32

\section{Preliminaries}

In this section, we recall some basic notions, and its properties that are needful for developing the main results.

Definition 2.1 [3] Let $(A, \rightarrow, *, 1)$ be an algebra with a complement “*” and a binary operation " $\rightarrow$ " is called a Wajsberg algebra (W-algebra) if and only if it satisfies the following axioms for all $x, y, z \in A$.

(i) $1 \rightarrow x=x$

(ii) $\quad(x \rightarrow y) \rightarrow((y \rightarrow z) \rightarrow(x \rightarrow z))=1$

(iii) $(x \rightarrow y) \rightarrow y=(y \rightarrow x) \rightarrow x$

(iv) $\left(x^{*} \rightarrow y^{*}\right) \rightarrow(y \rightarrow x)=1$

Proposition 2.2 [3] The $\mathrm{W}$-algebra $(A, \rightarrow, *, 1)$ satisfies the following equations and implications for all $x, y, z \in A$.

(i) $x \rightarrow x=1$

(ii) If $x \rightarrow y=y \rightarrow x=1$, then $x=y$

(iii) $x \rightarrow 1=1$

(iv) $x \rightarrow(y \rightarrow x)=1$

(v) If $x \rightarrow y=y \rightarrow z=1$, then $x \rightarrow z=1$

(vi) $\quad(x \rightarrow y) \rightarrow((z \rightarrow x) \rightarrow(z \rightarrow y))=1$

(vii) $x \rightarrow(y \rightarrow z)=y \rightarrow(x \rightarrow z)$

(viii) $x \rightarrow 0=x \rightarrow 1^{*}=x^{*}$

(ix) $\quad\left(x^{*}\right)^{*}=x$

(x) $\quad x^{*} \rightarrow y^{*}=y \rightarrow x$

Proposition 2.3 [3] The $\mathrm{W}$-algebra $(A, \rightarrow, *, 1)$ satisfies the following equations and implications for all $x, y, z \in A$.

(i) If $x \leq y$, then $x \rightarrow z \geq y \rightarrow z$

(ii) If $x \leq y$, then $z \rightarrow x \leq z \rightarrow y$

(iii) $x \leq y \rightarrow z$ if and only if $y \leq x \rightarrow z$

(iv) $\quad(x \vee y)^{*}=\left(x^{*} \wedge y^{*}\right)$

(v) $\quad(x \wedge y)^{*}=\left(x^{*} \vee y^{*}\right)$

(vi) $\quad(x \vee y) \rightarrow z=(x \rightarrow z) \wedge(y \rightarrow z)$

(vii) $\quad x \rightarrow(y \wedge z)=(x \rightarrow y) \wedge(x \rightarrow z)$

(viii) $\quad(x \rightarrow y) \vee(y \rightarrow x)=1$

(ix) $\quad x \rightarrow(y \vee z)=(x \rightarrow y) \vee(x \rightarrow z)$

(x) $\quad(x \wedge y) \rightarrow z=(x \rightarrow y) \vee(x \rightarrow z)$

(xi) $\quad(x \wedge y) \vee z=(x \vee z) \wedge(y \vee z)$

(xii) $\quad(x \wedge y) \rightarrow z=(x \rightarrow y) \rightarrow(x \rightarrow z)$

Definition 2.4 [3] The W-algebra $A$ is called a lattice $\mathrm{W}$-algebra if it satisfies the following conditions for all $x, y \in A$.

(i) A partial ordering $\leq$ on a lattice $\mathrm{W}$-algebra $A$ such that $x \leq y$ if and only if $x \rightarrow y=1$ 
B. A. Mohideen, A. Ibrahim / J. Math. Computer Sci. 9 (2014), 25-32

(ii) $\quad(x \vee y)=(x \rightarrow y) \rightarrow y$

(iii) $\quad(x \wedge y)=\left(\left(x^{*} \rightarrow y^{*}\right) \rightarrow y^{*}\right)^{*}$. Thus, we have $(A, \vee, \wedge, 0, *, 1)$ is a lattice $\mathrm{W}$-algebra with lower bound 0 and upper bound 1 .

Definition 2.5 [3] Let $A$ be W- algebra. A subset $F$ of $A$ is called an implicative filter of $A$ if it satisfies the following axioms for all $x, y \in A$.

(i) $1 \in F$

(ii) $x \in F$ and $x \rightarrow y \in F$ imply $y \in F$

Definition 2.6 [3] Let $A$ be W- algebra. A subset $F$ of $A$ is called a prime implicative filter of $A$ if it satisfies the axiom for all $x, y \in A,(x \vee y) \in F$ then $x \in F$ and $y \in F$.

Definition 2.7 [8] Let $X$ be a set. A function $\mu: X \rightarrow[0,1]$ is called a fuzzy subset on $X$, for each $x \in X$, the value of $\mu(x)$ describes a degree of membership of $x$ in $\mu$.

Definition 2.8 [8] Let $\mu$ be a fuzzy set in a set $X$. For $t \in[0,1]$, the set $\mu_{t}=\{x \in \mathrm{X} /$ $\mu(x) \geq t\}$ is called a level subset of $\mu$.

Definition 2.9 [1] A fuzzy subset $\mu$ of a lattice W- algebra $A$ is called a fuzzy implicative filter of $A$ if it satisfies the following

(i) $\mu(1) \geq \mu(x)$ for all $x \in A$

(ii) $\mu(y) \geq \min \{\mu(x \rightarrow y), \mu(x)\}$ for all $x, y \in A$

\section{Main results}

In this section, we introduce fuzzy prime and anti fuzzy prime implicative filters of lattice $\mathrm{W}$-algebras and investigate some properties with illustrations.

\subsection{Fuzzy prime filters}

Definition 3.1.1. A non-constant fuzzy implicative filter $\mu$ of a lattice $\mathrm{W}$ - algebra $A$ is said to be a prime if $\mu(x \vee y) \leq \max \{\mu(x), \mu(y)\}$ for all $x, y \in A$.

Example 3.1.2. Let $A=\{0, a, b, 1\}$ be a set with Figure (1) as partial ordering. Define a unary operation " $*$ " and a binary operation " $\rightarrow$ " on $A$ as in the Tables (1) and (2).

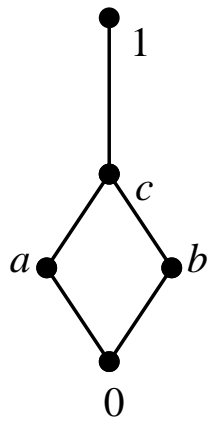

Figure (1)

\begin{tabular}{|l|l|}
\hline$x$ & $x^{*}$ \\
\hline 0 & 1 \\
$a$ & $b$ \\
$b$ & $a$ \\
$c$ & $c$ \\
1 & 0 \\
\hline
\end{tabular}

Table (1)

\begin{tabular}{|c|ccccc|}
\hline$\rightarrow$ & 0 & $a$ & $b$ & $c$ & 1 \\
\hline 0 & 1 & 1 & 1 & 1 & 1 \\
$a$ & $b$ & 1 & $c$ & 1 & 1 \\
$b$ & $a$ & $c$ & 1 & 1 & 1 \\
$c$ & $c$ & $c$ & $c$ & 1 & 1 \\
1 & 0 & $a$ & $b$ & $c$ & 1 \\
\hline
\end{tabular}

Table (2)

Define $\vee$ and $\wedge$ an operation on $A$ as follows: $(x \vee y)=(x \rightarrow y) \rightarrow y$, 
$(x \wedge y)=\left(\left(x^{*} \rightarrow y^{*}\right) \rightarrow y^{*}\right)^{*}$ for all $x, y \in A$. Then, we have $(A, \vee, \wedge, 0,1)$ is a lattice $\mathrm{W}$ - algebra. Consider the fuzzy subset $\mu$ on $A$ is defined by

$$
\mu(x)=\left\{\begin{array}{l}
0.8 \text { if } x \in\{1\} \\
0.6 \text { if } x \in\{0, a, b, c\}
\end{array} \text { for all } x \in A .\right.
$$

Then $\mu$ is a fuzzy prime implicative filter of $A$.

Example 3.1.3. Let $A=\{0, a, b, c, d, 1\}$ be a set with Figure (2) as partial ordering. Define a unary operation " $*$ " and a binary operation " $\rightarrow$ " on $A$ as in the Table (3) and Table (4)

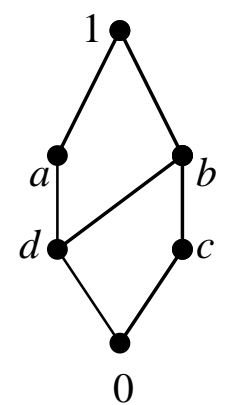

Figure (2)

\begin{tabular}{|c|c|}
\hline$x$ & $x^{*}$ \\
\hline 0 & 1 \\
$b$ & $c$ \\
$b$ & $d$ \\
$c$ & $a$ \\
$d$ & $b$ \\
1 & 0 \\
\hline
\end{tabular}

Table (3)

\begin{tabular}{|l|llllll|}
\hline$\rightarrow$ & 0 & $a$ & $b$ & $c$ & $d$ & 1 \\
\hline 0 & 1 & 1 & 1 & 1 & 1 & 1 \\
$a$ & $c$ & 1 & $b$ & $c$ & $b$ & 1 \\
$b$ & $d$ & $a$ & 1 & $b$ & $a$ & 1 \\
$c$ & $a$ & $a$ & 1 & 1 & $a$ & 1 \\
$d$ & $b$ & 1 & 1 & $b$ & 1 & 1 \\
1 & 0 & $a$ & $b$ & $c$ & $d$ & 1 \\
\hline
\end{tabular}

Table (4)

Define $\vee$ and $\wedge$ an operation on $A$ as follows:

$(x \vee y)=(x \rightarrow y) \rightarrow y$,

$(x \wedge y)=\left(\left(x^{*} \rightarrow y^{*}\right) \rightarrow y^{*}\right)^{*}$ for all $x, y \in A$. Then, we have $(A, \vee, \wedge, 0,1)$ is a lattice $\mathrm{W}$ - algebra.

Consider a fuzzy subset $\mu$ on $A$ is defined by

$$
\mu(x)=\left\{\begin{array}{l}
0.5 \text { if } x \in\{1, a, b\} \\
0.3 \text { if } x \in\{0, c, d\}
\end{array} \quad \text { for all } x \in A .\right.
$$

Then, we have $\mu$ is not a fuzzy prime implicative filter of lattice $\mathrm{W}$-algebra $A$.

Since $\mu(c \vee d)=0.5$. But, $\max \{\mu(c), \mu(d)\}=0.3$.

Thus, we get $\mu(c \vee d) \not \leq \max \{\mu(c), \mu(d)\}$.

Proposition 3.1.4. If $\mu$ is a fuzzy implicative filter of lattice $\mathrm{W}$ - algebra $A$. Then the set $A_{\mu}=\{x \in A / \mu(x)=\mu(1)\}$ is a implicative filter of $A$.

Proof. Let $\mu$ be a fuzzy implicative filter of lattice W-algebra $A$.

Consider the set $A_{\mu}=\{x \in A / \quad \mu(x)=\mu(1)\}$.

Then, for any $x \in A \Rightarrow \mu(x) \in A_{\mu} \Rightarrow \mu(1) \in A_{\mu}$.

If $(\mu(x) \rightarrow \mu(1)) \in A_{\mu}$.

$$
\begin{aligned}
& \Rightarrow(\mu(x) \rightarrow \mu(1))=1 \\
& \Rightarrow(\mu(x) \leq \mu(y)), \text { since } x \leq y \text { if and only if } x \rightarrow y=1 \\
& \Rightarrow(\mu(1) \leq \mu(y)) .
\end{aligned}
$$

Since $\mu$ is fuzzy implicative filter of lattice W-algebra, $(\mu(1) \geq \mu(y))$. 
From (1) and (2), we have $\mu(y)=\mu(1)$. Thus, $\mu(y) \in A_{\mu}$. Hence, we get $A_{\mu}$ is a implicative filter of lattice $\mathrm{W}$ - algebra $A$.

Proposition 3.1.5. Let $\mu$ be a fuzzy prime implicative filter of lattice $\mathrm{W}$ - algebra $A$. Then the set $A_{\mu}=\{x \in A / \mu(x)=\mu(1)\}$ is a prime implicative filter of $A$.

Proof. We have, $A_{\mu}$ is a implicative filter of $A$ (by Proposition 3.1.4).

For any $x, y \in A$, we have $(x \vee y) \in A_{\mu}$.

Then, we have $\mu(1)=\mu(x \vee y) \leq \max \{\mu(x), \mu(y)\}=\mu(x)$ or $\mu(y)$.

Thus, $\mu(x)=\mu(1)$ or $\mu(y)=\mu(1)$. Hence, we get $\mu(x) \in A_{\mu}$ or $\mu(y) \in A_{\mu}$.

Therefore, $A_{\mu}$ is a prime implicative filter of $A$.

Proposition 3.1.6. A fuzzy subset $\mu$ is a fuzzy prime implicative filter of lattice $\mathrm{W}$ algebra $A$, if and only if the set $S=\{x \in A / \mu(x)=1\}$ is either empty or a fuzzy prime implicative filter of $A$.

Proof. Let $\mu$ be a fuzzy prime implicative filter of lattice $\mathrm{W}$ - algebra $A$.

Then, we get $\mu(x \vee y) \leq \max \{\mu(x), \mu(y)\}$ for all $x, y \in A$.

Consider the set $S=\{x \in A / \mu(x)=1\}$.

If $S \neq \phi$. We have to prove that $S$ is a fuzzy prime implicative filter of $A$.

Since $S \neq \phi$, for any $x, y \in A$ and $\mu(x), \mu(y) \in S$ such that $\mu(x)=1$ and $\mu(y)=1$.

Now $\mu(x \vee y) \leq \max \{\mu(x), \mu(y)\}=1$.

Thus, we have $\mu(x \vee y) \in S$ for all $x, y \in A$.

Hence, $S$ is a fuzzy prime implicative filter of $A$.

Conversely, if $S \neq \phi$ and $S$ is a fuzzy prime implicative filter of $A$.

To Prove: Fuzzy subset $\mu$ is a fuzzy prime implicative filter of $A$.

For any $x, y \in A$ and $\mu(x), \mu(y) \in S$ such that $\mu(x)=1$ and $\mu(y)=1$.

Now $\mu(x \vee y)=1$ and $\max \{\mu(x), \mu(y)\}=1$.

Thus, we get $\mu(x \vee y) \leq \max \{\mu(x), \mu(y)\}$ for all $x, y \in A$.

Hence, $\mu$ is a fuzzy prime implicative filter of lattice $\mathrm{W}$ - algebra $A$.

Proposition 3.1.7. Let $F$ be a implicative filter of a lattice $\mathrm{W}$ - algebra $A$, and let $\alpha<\beta$ $\neq 0$ be elements of $[0,1)$. Then fuzzy set $\mu: A \rightarrow[0,1]$ defined by

$$
\mu(x)=\left\{\begin{array}{l}
\beta \text { if } x \in F \\
\alpha \text { otherwise }
\end{array} \text { for all } x \in A, \text { is a fuzzy implicative filter of } A .\right.
$$

Proof. Since $1 \in F, \mu(1)=\beta \geq \mu(x)$ for all $x \in A$.

Suppose $\mu(y) \geq \min \{\mu(x \rightarrow y), \mu(x)\}$ for all $x, y \in A$ does not hold.

Then, there exists $x, y \in A$, such that $\mu(x)=\alpha$ and $\min \{\mu(x \rightarrow y), \mu(x)\}=\beta$.

Thus, we have $\mu(x \rightarrow y)=\beta$ and $\mu(x)=\beta$. Hence, $x \rightarrow y \in F$ and $x \in F$ and so $y \in F$, since $F$ is a implicative filter. This is a contradiction. Therefore, $\mu(x)$ is a fuzzy implicative filter of $A$.

Proposition 3.1.8. Let $S$ be a prime implicative filter of lattice $\mathrm{W}$ - algebra $A$ and let $\alpha \in[0,1]$. If $\mu$ is a fuzzy subset in $A$ defined by $\mu(x)=\left\{\begin{array}{l}1 \text { if } x \in S \\ \alpha \text { otherwise }\end{array}, \forall x \in A\right.$. (3)

Then, $\mu$ is a fuzzy prime implicative filter of $A$. 
Proof. By the result (Proposition 3.1.7), we have $\mu$ is a fuzzy implicative filter of $A$. For any $x, y \in A$, we have $(x \vee y) \in S$ then $x \in S$ or $y \in S$.

Thus, $\mu(x \vee y)=1=\max \{\mu(x), \mu(y)\}$.

If $(x \vee y) \notin S$ then $\mu(x \vee y)=\alpha \leq \max \{\mu(x), \mu(y)\}$.

From (4) and (5), we have $\mu$ is a fuzzy prime implicative filter of $A$.

Corollary 3.1.9. If $S$ is a prime implicative filter of lattice $\mathrm{W}$ - algebra $A$, then the characteristic function $\chi_{S}$ is a fuzzy prime implicative filter of $A$.

Now we see the converse of Corollary 3.1.9.

Proposition 3.1.10. If $S$ is a filter of lattice $\mathrm{W}$-algebra $A$ such that the characteristics function $\chi_{S}$ is a fuzzy prime implicative filter of $A$, then $S$ is a prime implicative filter of $A$.

Proof. Let $x, y \in A$ be such that $(x \vee y) \in S$ and $x \notin S$.

Then, we get $1=\chi_{s}(x \vee y)$, by (3) of the Proposition 3.1.8.

$$
\leq \max \left\{\chi_{s}(x), \chi_{s}(y)\right\}=\chi_{s}(y) \text {. }
$$

Thus, we have $\chi_{S}(y)=1$ so that $y \in S$. Hence, $S$ is a prime filter of $A$.

\subsection{Anti fuzzy prime filters}

Definition 3.2.1. A non-constant fuzzy implicative filter of lattice $\mathrm{W}$ - algebra $A$ is called an anti fuzzy prime if $\mu(x \wedge y) \geq \min \{\mu(x), \mu(y)\}$ for all $x, y \in A$.

Example 3.2.2. Let $A=\{0, a, b, c, 1\}$ be a set with Figure (3) as partial ordering. Define unary operation " $*$ " and a binary operation " $\rightarrow$ " on $A$ as in the Table (5) and Table (6)

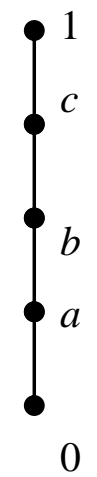

Figure (3)

\begin{tabular}{|l|l|}
\hline$x$ & $x^{*}$ \\
\hline 0 & 1 \\
$a$ & $c$ \\
$b$ & $b$ \\
$c$ & $a$ \\
1 & 0 \\
\hline
\end{tabular}

Table (5)

\begin{tabular}{|l|lllll|}
\hline$\rightarrow$ & 0 & $a$ & $b$ & $c$ & 1 \\
\hline 0 & 1 & 1 & 1 & 1 & 1 \\
$a$ & $c$ & 1 & 1 & 1 & 1 \\
$b$ & $b$ & $c$ & 1 & 1 & 1 \\
$c$ & $a$ & $b$ & 1 & 1 & 1 \\
1 & 0 & $a$ & $b$ & $c$ & 1 \\
\hline
\end{tabular}

Table (6)

Define $\vee$ and $\wedge$ an operation on $A$ as follows: $(x \vee y)=(x \rightarrow y) \rightarrow y$, $(x \wedge y)=\left(\left(x^{*} \rightarrow y^{*}\right) \rightarrow y^{*}\right)^{*}$ for all $x, y \in A$.

Then, we have $(A, \vee, \wedge, 0,1)$ is a lattice $\mathrm{W}$ - algebra.

Consider the fuzzy subset $\mu$ on $A$ is defined by

$$
\mu(x)=\left\{\begin{array}{l}
0.6 \text { if } x=1 \\
0.3 \text { otherwise }
\end{array} \text { for all } x \in A .\right.
$$

Then $\mu$ is a anti fuzzy prime implicative filter of $A$. 
Proposition 3.2.3. Let $\mu$ be an anti fuzzy prime implicative filter of lattice $\mathrm{W}$ - algebra $A$. Then the set $A_{\mu}=\{x \in A / \mu(x)=\mu(1)\}$ is a prime implicative filter of $A$.

Proof. We have, $A_{\mu}$ is a implicative filter of $A$ (by Proposition 3.1.4)

Let $x, y \in A$ be such that $(x \wedge y) \in A_{\mu}$.

Then, we have $\mu(1)=\mu(x \wedge y) \geq \min \{\mu(x), \mu(y)\}=\mu(x)$ and $\mu(y)$.

If $\mu(x)=\mu(1), \mu(y)=\mu(1)$, then $\mu(x) \in A_{\mu}$ and $\mu(y) \in A_{\mu}$.

Therefore, $A_{\mu}$ is a prime implicative filter of $A$.

Proposition 3.2.4. A fuzzy subset $\mu$ is an anti fuzzy prime implicative filter of lattice W- algebra $A$, if and only if the set $T=\{x \in A / \mu(x)=1\}$ is either empty or a fuzzy prime implicative filter of $A$.

Proof. Let $\mu$ be a anti fuzzy prime implicative filter of lattice $\mathrm{W}$ - algebra $A$. Then, we get $\mu(x \wedge y) \geq \min \{\mu(x), \mu(y)\}$ for all $x, y \in A$.

Consider the set $T=\{x \in A / \mu(x)=1\}$. If $T \neq \phi$. We have to show that $T$ is a fuzzy prime implicative filter of $A$. Since $T \neq \phi$, for any $x, y \in A$ and $\mu(x), \mu(y) \in T$ such that $\mu(x)=1$ and $\mu(y)=1$.Now $\mu(x \wedge y) \geq \min \{\mu(x), \mu(y)\}=1$. Thus, we have $\mu(x \wedge y) \in T$ for all $x, y \in A$. Hence, $T$ is a fuzzy prime implicative filter of $A$. Conversely, if $T \neq \phi$ then $T$ is a fuzzy prime implicative filter of $A$.

For any $x, y \in A$ and $\mu(x), \mu(y) \in T$ such that $\mu(x)=1$ and $\mu(y)=1$.

Now $\mu(x \wedge y)=1$ and $\min \{\mu(x), \mu(y)\}=1$.

Thus, we get $\mu(x \wedge y) \geq \min \{\mu(x), \mu(y)\}$ for all $x, y \in A$.

Hence, $\mu$ is an anti fuzzy prime implicative filter of lattice $\mathrm{W}$ - algebra $A$.

Proposition 3.2.5. Let $I$ be a prime implicative filter of a lattice $\mathrm{W}$-algebra $A$, let $\alpha<\beta \neq 0$ be the elements of $(0,1]$. Then fuzzy set $\mu: A \rightarrow[0,1]$ defined by

$$
\mu(x)=\left\{\begin{array}{ll}
\alpha & \text { if } x \in I \\
\beta & \text { otherwise }
\end{array} \text { for all } x \in A \text { is an anti fuzzy implicative filter of } A .\right.
$$

Proof. Since $1 \in I, \mu(1)=\alpha \geq \mu(x)$ for all $x \in A$.

Suppose $\mu(y) \geq \min \{\mu(x \rightarrow y), \mu(x)\}$, for all $x, y \in A$ does not hold.

Then there exists $x, y \in A$ such that $\mu(x)=\beta$ and $\min \{\mu(x \rightarrow y), \mu(x)\}=\alpha$.

Thus, we have $\mu(x \rightarrow y)=\alpha$ and $\mu(x)=\alpha$.

Therefore, $x \rightarrow y \in I$ and $x \in I$ so $y \in I$, since $I$ is a implicative filter.

This is a contradiction. Hence, $\mu(x)$ is a anti fuzzy implicative filter of $A$.

Proposition 3.2.6. Let $T$ be a prime implicative filter of lattice $\mathrm{W}$ - algebra $A$ and let $\alpha \in(0,1]$. If $\mu$ is a fuzzy subset in $A$ defined by

$$
\mu(x)=\left\{\begin{array}{ll}
1 & \text { if } x \in T \\
\alpha & \text { otherwise }
\end{array} \text { for all } x \in A\right.
$$

Then $\mu$ is an anti fuzzy prime implicative filter of $A$.

Proof. By the result (Proposition 3.2.5), we have $\mu$ is an anti fuzzy implicative filter of $A$. For any $x, y \in A$, we have $(x \wedge y) \in T$ then, $x \in T$ and $y \in T$.

Thus, $\mu(x \wedge y)=1=\min \{\mu(x), \mu(y)\}$. 
If $(x \wedge y) \notin S$, then $\mu(x \wedge y)=\alpha \geq \min \{\mu(x), \mu(y)\}$.

From (7) and (8), we have $\mu$ is a anti fuzzy prime implicative filter of $A$.

Proposition 3.2.7. Let $T$ be a filter of lattice W- algebra $A$. Then $T$ is a prime implicative filter of $A$ if and only if the complement $\chi_{s}{ }^{*}$ of the characteristics functions is an anti fuzzy prime implicative filter of $A$.

Proof. Obviously, If $T$ is a prime implicative filter of lattice $\mathrm{W}$-algebra $A$, then the characteristics function $\chi_{s}{ }^{*}$ is an anti fuzzy prime implicative filter of $A$.

Conversely, the complement $\chi_{S}{ }^{*}$ of characteristic function is an anti fuzzy prime implicative filter of $A$, we have to prove that $T$ is a prime implicative filter of $A$.

Suppose $(x \wedge y) \in T$ and $x \notin T$ for all $x, y \in A$.

Then, $1=\chi_{s}^{*}(x \wedge y)=1-\chi_{s}(x \wedge y)$, by (6) of Proposition 3.2.6.

$$
\begin{aligned}
& \geq 1-\min \left\{\chi_{S}(x), \chi_{S}(y)\right\} \\
& =\max \left\{1-\chi_{S}(x), 1-\chi_{S}(y)\right\} \\
& =\max \left\{\chi_{S}{ }^{*}(x), \chi_{S}{ }^{*}(y)\right\} \\
& =\chi_{S}{ }^{*}(y) .
\end{aligned}
$$

Thus, we have $\chi_{S}{ }^{*}(y)=1$. Therefore, $y \in T$. Hence, we have $T$ is a prime implicative filter of $A$.

\section{Conclusion}

In this paper, we have shown that $A_{\mu}$ is implicative filter in which $\mu$ is fuzzy implicative filter of lattice $\mathrm{W}$-algebra $A$. By using this result, we have proved that $A_{\mu}$ is prime implicative filter of $A$. Further, we have discussed some properties of fuzzy prime implicative filter, and investigated that the set of anti fuzzy prime implicative filter is either empty or fuzzy prime implicative filter. Finally, we have given that the complement characteristic function is anti fuzzy prime implicative filter of lattice $\mathrm{W}$ algebra $A$.

\section{Acknowledgements}

The authors would like to thank the Editor-in Chief, editorial board members and referees for their valuable suggestions and comments.

\section{References}

[1] M. Basheer Ahamed and A. Ibrahim, Fuzzy implicative filters of lattice Wajsberg Algebras, Advances in Fuzzy Mathematics, 6 (2) (2011) 235-243.

[2] M. Basheer Ahamed, and A. Ibrahim, Anti fuzzy implicative filters in lattice W-algebras, International journal of Computational Science and Mathematics, 4 (1) (2012) 49-56.

[3] J. M. Font, A. J. Rodriguez and A. Torrens, Wajsberg Algebras, STOCHASTICA Vol. VIII, No.1 (1984), $5-31$.

[4] Eshagh Hosseinpour, T-Rough Fuzzy Subgroups of Groups, Journal of Mathematics and Computer Science 12 (2014) 186-195.

[5] Y. Imai and K. Iseki, On axiom systems of propositional calculi, Proc. Japan Academy, 42 (1966) 19-22.

[6] A. Rose, and J. B Rosser, Fragments of many-valued statement calculi, Transactions of the American Mathematical Society 87 (1958) 1-53.

[7] O. G. Xi, Fuzzy BCK-algebras, Math. Japon. 36 (1991) 935-942.

[8] L. A. Zadeh, Fuzzy sets, Information and Control 8 (1965) 338-353. 